\title{
Transcriptome-Based Identification of a Functional Fasciola hepatica Carboxylesterase B
}

\author{
Yaretzi J. Pedroza-Gómez ${ }^{1}$, Raquel Cossio-Bayugar ${ }^{1}$, Hugo Aguilar-Díaz ${ }^{1}$, Silvana Scarcella ${ }^{2}$, \\ Enrique Reynaud $^{3}$ (D), María del Rayo Sanchez-Carbente ${ }^{4}$, Verónica Narváez-Padilla ${ }^{5}$ (D) \\ and Estefan Miranda-Miranda $1, *$ (D)
}

Citation: Pedroza-Gómez, Y.J.; Cossio-Bayugar, R.; Aguilar-Díaz, H. Scarcella, S.; Reynaud, E.; Sanchez-Carbente, M.d.R.; Narváez-Padilla, V.;

Miranda-Miranda, E. TranscriptomeBased Identification of a Functional Fasciola hepatica Carboxylesterase B. Pathogens 2021, 10, 1454. https:// doi.org/10.3390/pathogens10111454

Academic Editors: Jorge Hugo Calvo and Inmaculada Martín-Burriel

Received: 13 September 2021

Accepted: 5 November 2021

Published: 10 November 2021

Publisher's Note: MDPI stays neutral with regard to jurisdictional claims in published maps and institutional affiliations.

Copyright: (C) 2021 by the authors. Licensee MDPI, Basel, Switzerland. This article is an open access article distributed under the terms and conditions of the Creative Commons Attribution (CC BY) license (https:// creativecommons.org/licenses/by/ $4.0 /)$
1 Centro Nacional de Investigaciones Disciplinarias en Salud Animal e Inocuidad, Instituto Nacional de Investigaciones Forestales Agrícolas y Pecuarias (INIFAP), Boulevard Cuauhnahuac No. 8534, Jiutepec 62550, Morelos, Mexico; yaretzijpg@gmail.com (Y.J.P.-G.); cossio.raquel@inifap.gob.mx (R.C.-B.); aguilar.hugo@inifap.gob.mx (H.A.-D.)

2 Facultad de Ciencias Veterinarias, Campus Universitario, Universidad Nacional del Centro de la Provincia de Buenos Aires, Tandil 7000, Argentina; silvanas@vet.unicen.edu.ar

3 Departamento de Genética del Desarrollo y Fisiología Molecular, Instituto de Biotecnología, Universidad Nacional Autónoma de México, Avenida Universidad, 2001, Apartado Postal 510-3, Cuernavaca 62210, Morelos, Mexico; enrique@ibt.unam.mx

4 Centro de Investigación en Biotecnología de la Universidad Autónoma del Estado de Morelos, Av. Universidad 1001, Cuernavaca 62209, Morelos, Mexico; maria.sanchez@uaem.mx

5 Centro de Investigación en Dinámica Celular de la Universidad Autónoma del Estado de Morelos, Cuernavaca Morelos México, Av. Universidad 1001, Chamilpa, Cuernavaca 62210, Morelos, Mexico; vaezgm@gmail.com

* Correspondence: miranda.estefhan@inifap.gob.mx; Tel.: +52-77-7319-2860 (ext. 119)

\begin{abstract}
Bioinformatics analysis of the complete transcriptome of Fasciola hepatica, identified a total of ten putative carboxylesterase transcripts, including a $3146 \mathrm{bp}$ mRNA transcript coding a $2205 \mathrm{bp}$ open reading frame that translates into a protein of 735 amino acids, resulting in a predicted protein mass of $83.5 \mathrm{kDa}$ and a putative carboxylesterase $\mathrm{B}$ enzyme. The gene coding for this enzyme was found in two reported $F$. hepatica complete genomes stretching $23,230 \mathrm{bp}$, containing two exons of 1282 and $1864 \mathrm{bp}$, respectively, as well as a 20,084 bp intron between the exons. The enzymatic activity was experimentally assayed on $F$. hepatica protein extracts by SDS-PAGE zymograms using synthetic chromogenic substrates, confirming both the theoretical molecular weight and carboxylesterase enzymatic activity. Further bioinformatics predicted that this enzyme is an integral component of the cellular membrane that should be active as a $167 \mathrm{kDa}$ homodimer complex and polyacrylamide gel electrophoresis (PAGE) zymograms experiments confirmed the analysis. Additional bioinformatics analysis showed that DNA sequences that code for this particular enzyme are highly conserved in other parasitic trematodes, although they are labeled hypothetical proteins.
\end{abstract}

Keywords: Fasciolosis; bioinformatics; transcriptome; carboxylesterase; zymogram

\section{Introduction}

The liver fluke Fasciola hepatica is a worldwide zoonotic parasitic trematode affecting a vast range of animal hosts, including humans. It is the causative agent of fascioliasis, a parasitic disease highly prevalent in the livestock industry and capable of parasitizing millions of people worldwide [1]. Digenetic trematodes such as F. hepatica require a gastropod mollusk as an intermediate host to complete a complex biological cycle; for this reason, fascioliasis distribution is closely related to the intermediate host distribution worldwide [2].

The prevention of fascioliasis is achieved by the use of anthelmintics on the livestock, a procedure that sets the conditions for anthelmintic resistance selection in the liver fluke populations [3-5]. F. hepatica is an example of the ever-growing resistance to the effects of 
antiparasitic compounds such as benzimidazole-derived anthelmintics. Previous research on the subject suggests that several xenobiotic metabolizing enzymes (XMEs) may be associated with the resistance phenomenon [6]. Xenobiotic metabolism is responsible for the defense of most organisms against poisonous effects of naturally occurring toxins and the enzymes responsible for this type of metabolism are the focus of scientific scrutiny because they mediate the ever-increasing tolerance and resistance of parasites against antiparasitic agents that plague livestock and humans [7].

Carboxylesterases are a family of XMEs found in all types of organisms [8]; these enzymes hydrolyze xenobiotic compounds bearing chemical functional groups such as carboxylic acid ester, amide and thioester into products containing alcohols and organic acids that then become easily disposable by other supplementary metabolic pathways [9].

Carboxylesterases are implicated in diverse biological functions, in addition to their role in the detoxification of xenobiotics, most notably juvenile hormone metabolism, which regulates the development of arthropods and other invertebrates [10].

The expression and enzymatic specific activity of carboxylesterases have been demonstrated to play a significant role in the resistance mechanism of a variety of parasites against antiparasitic compounds, such as the Plasmodium falciparum response against antimalarial compounds [11,12], insecticide resistance in the malaria vector Anopheles funestus [13], malathion resistance in the triatomid vector of American trypanosomiasis Rhodnius prolixus [14], and organophosphate resistance in the oriental fruit fly Bactrocella dorsalis [15]. Scientific evidence on carboxylesterases associated with insecticide resistance made this $\mathrm{XME}$ a reliable molecular marker for resistance screening in populations of the encephalitis vector, the Florida SLE mosquito Culex nigripalpus [16].

Although there is no previous study of F. hepatica carboxylesterase enzymatic activity that may demonstrate a probable linkage to anthelmintic resistance, it has been reported that carboxylesterase enzymatic specific activity in F. hepatica is increased in adult parasites recovered from anthelmintic-treated sheep hosts [17], during this study, some basic biochemical properties of F. hepatica carboxylesterase, such as the mass and catalytic activity on synthetic chromogenic substrates, were identified for the first time using zymogram assays on cytosolic protein extracts [17]. However, the lack of information on the DNA sequence of this particular gene represents an obstacle to understanding the complementary molecular properties of this enzyme or its probable role in the metabolism of anthelmintics [18]. Consequently, it is the objective of this study is to identify F. hepatica carboxylesterase mRNA transcript sequences using high-throughput transcriptome sequencing of total mRNA of the parasite. Once located, a comprehensive bioinformatics analysis of the gene DNA sequence features and its mRNA transcript as well as the properties of the predicted expressed protein is performed and, finally, the predicted proteomic results are compared to experimentally obtained biochemical properties from the actual enzyme found in parasite protein extracts.

\section{Results}

\subsection{Carboxylesterase Enzymatic Assays and Zymograms}

(1) Carboxylesterase enzymatic activity on synthetic chromogenic substrate, demonstrated the presence of high levels of enzymatic specific activity in three different parasite extracts, showing 3.1, 8.3 and $4.7 \mu \mathrm{mol} / \mathrm{mg} / \mathrm{min}$ hydrolyzed substrate for the crude extract, membrane fraction and soluble fraction, respectively (Figure 1).

(2) The SDS-PAGE zymograms showed two isozymes exhibiting carboxylesterasespecific activity as bands of 85 and $170 \mathrm{kDa}$ in the extract's cytosolic supernatant, whereas only a $170 \mathrm{kDa}$ band was found in the membrane fraction of the protein extract; these data and zymogram are represented in Figure 1. 


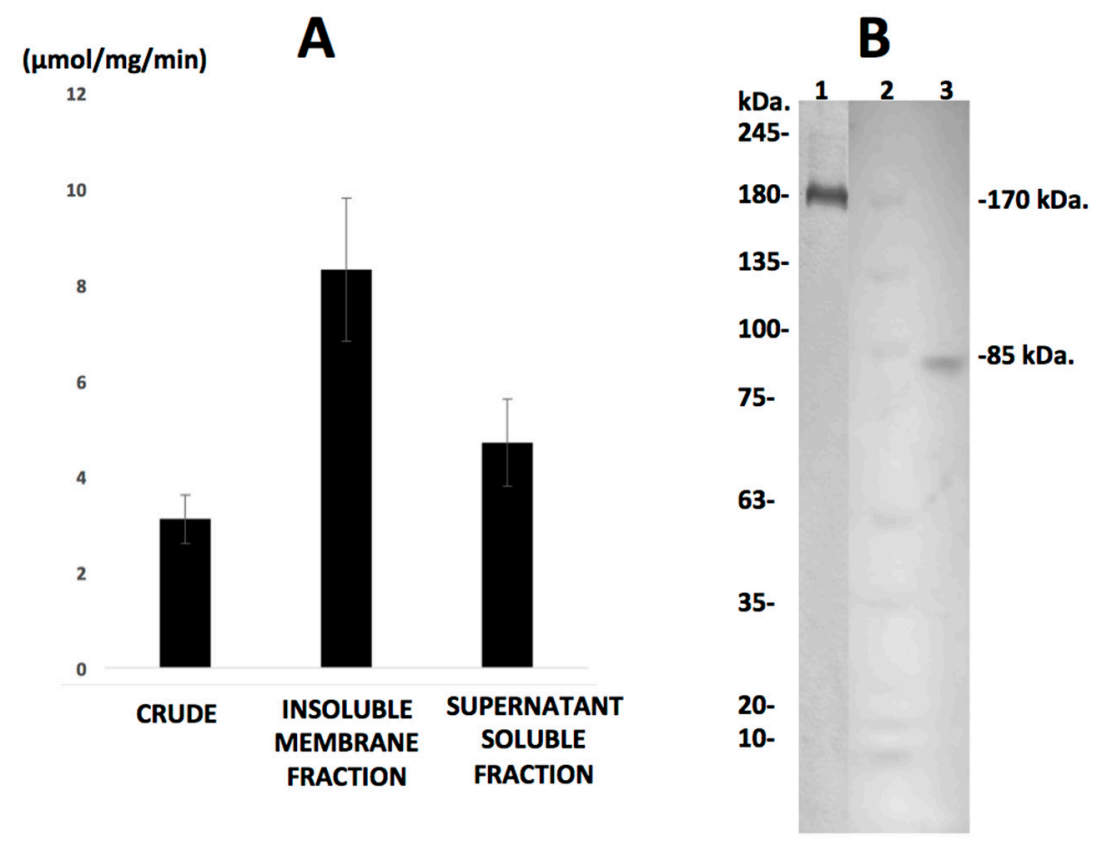

Figure 1. Fasciola hepatica Carboxyl esterase specific activity and SDS-PAGE zymograms. (A): Enzyme-specific activity from parasite's protein extracts measured by hydrolysis of the chromogenic synthetic substrate $\alpha$-naphtyl acetate coupled to the diazonium salt Fast Gardner at $524 \mathrm{~nm}$. (B): Non-reducing SDS-PAGE zymograms of the insoluble membrane fraction (lane 1) and the soluble fraction (lane 3), a carboxylesterase enzyme of $85 \mathrm{kDa}$ is detected in the soluble fraction whereas, a $170 \mathrm{kDa}$ enzyme was found in the membrane fraction. Lane 2 contains a commercial pre-stained protein molecular mass reference.

\subsection{F. hepatica Transcriptome}

(1) The F. hepatica transcriptome database obtained 15530 transripts made public at GenBank BioProject PRJNA679050, BioSample SAMN16822858, RNAseq data SRR13076124 available on-line at https:/ / trace.ncbi.nlm.nih.gov/Traces/sra/?run=SRR13076124. (accessed on 8 November 2021)

(2) The transcriptome sequencing data identified ten transcripts labeled esterase by Gene Ontology/Orthology, and two additional sequences tagged as esterase were not represented in our transcriptome but were found in the F. hepatica complete genome datasets available at the GenBank and WormBase-ParaSite databases and described at Table 1.

(3) The differential expression levels in fragments per million kilobases (FPMKs) from every esterase in our transcriptome determined during the sequencing procedure, were annotated in Table 1 and graphically represented in Figure 2. 
Table 1. mRNA transcripts with predicted esterase enzymatic specific activity. The F. hepatica transcriptome made public as RNAseq data SRR13076124 available on-line at https:/ / trace.ncbi.nlm.nih.gov / Traces/sra/?run=SRR13076124 (accessed on 8 November 2021), identified ten transcripts labeled esterase by Gene Ontology/Orthology, and two additional sequences tagged as esterase were not represented in our transcriptome but were found in the F. hepatica complete genome datasets available at the GenBank and WormBase-ParaSite databases.

\begin{tabular}{|c|c|c|c|c|c|c|c|}
\hline $\begin{array}{c}\text { Transcript } \\
\text { SRR13076124 } \\
\text { Spot No. }\end{array}$ & $\begin{array}{l}\text { Size } \\
\text { bp }\end{array}$ & AA & $\begin{array}{l}\text { Molecular } \\
\text { Mass }\end{array}$ & $\begin{array}{l}\text { Expression } \\
\text { FPKM }\end{array}$ & Description & $\begin{array}{c}\text { Genome Identity } \\
\text { UNIPROTKB/Description }\end{array}$ & $\begin{array}{c}\text { Genome Identity } \\
\text { GenBank/Description }\end{array}$ \\
\hline 473 & 3146 & 735 & 83.5 & 14.45 & Carboxylesterase B & A0A4E0S0J7/Coesterase & D915-000180/Hypotetical \\
\hline 12214 & 1837 & 424 & 25.41 & 0.42 & Carboxylesterase B & No match & $\begin{array}{l}\text { KZ430433.1/ Juvenile } \\
\text { hormone esterase }\end{array}$ \\
\hline 4519 & 3482 & 697 & 78.8 & 4.11 & Cholinesterase & AOAA4E0RPG5/Neuroligin & THD27534.1/Cholinesterase \\
\hline 4520 & 1816 & 383 & 44.0 & 2.1 & Acetylcholinesterase & $\begin{array}{l}\text { AOAA4E0RPG/Carboxylic ester } \\
\text { hydrolase }\end{array}$ & THD27534/Cholinesterase \\
\hline 12214 & 1837 & 430 & 48.4 & 1.4 & $\begin{array}{l}\text { Carboxylic Ester } \\
\text { Hydrolase }\end{array}$ & $\begin{array}{c}\text { A0A4E0S092 } \\
\text { /Uncharacterized protein }\end{array}$ & D915_006009/Hypotetical \\
\hline 4519 & 2094 & 697 & 78.8 & 1.2 & Neuroligin & $\begin{array}{c}\text { D915_001711/Carboxylic ester } \\
\text { hydrolase }\end{array}$ & THD27534/Cholinesterase \\
\hline 1986 & 1424 & 502 & 56.9 & 0.7 & Cholinesterase & $\begin{array}{l}\text { A0A2H1CBM8 } \\
\text { Cholinesterase }\end{array}$ & $\begin{array}{l}\text { PIS84875.1 } \\
\text { /Neuroligin }\end{array}$ \\
\hline 249 & 2085 & 694 & 79.7 & 1.5 & Neurotactin & A0A4E0RJZ9/Neurotactin & THD28606.1/Neurotactin \\
\hline 247 & 1510 & 490 & 56.19 & 2.6 & Cholinesterase & A0A2H1CVT6/ Cholinesterase & THD28606/Neurotactin \\
\hline 2545 & 2304 & 606 & 69.2 & 0.7 & Uncharacterized & 0A2H1CVW9/ Uncharacterized & $\begin{array}{l}\text { THD28213.1/Protein } \\
\text { Notum }\end{array}$ \\
\hline No match & 1934 & 644 & 72.7 & No data & Cholinesterase & A0A2H1CPF2/ Carboxylesterase & PIS89339/ Cholinesterase \\
\hline No match & 1689 & 562 & 63.1 & No data & Carboxylesterase & No match & $\begin{array}{c}\text { KZ429703.1/ } \\
\text { Carboxylesterase }\end{array}$ \\
\hline
\end{tabular}

\section{Differential expression}

FPMK

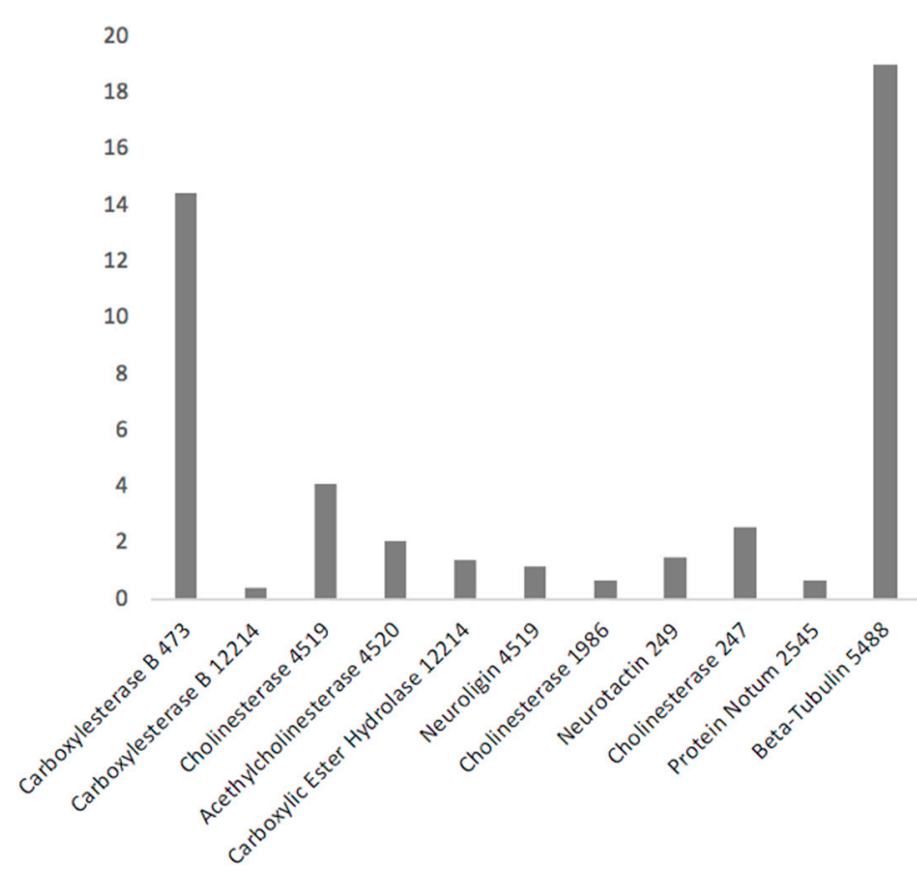

Figure 2. Differential expression of mRNA transcripts with predicted esterase enzymatic specific activity. The differential expression levels in fragments per million kilobases (FPMKs) from every esterase in our transcriptome were determined during the sequencing procedure, annotated in Table 1 and graphically represented. Gene onthology predicted function and transcript number at the SRR13076124 database were included at the footnote for every transcript. The constitutive gen Beta-Tubulin found in our transcriptome as transcript 5488, was used as high expression reference. 


\subsection{Bioinformatics Analysis}

(1) All transcripts sequences identified as esterase by the gene ontology enrichment analysis were compared by differential expression to the constitutive gene BetaTubulin(14.45 FPMKs) as well as the mRNA size required to translate into an $85 \mathrm{kDa}$ protein or a homodimer complex of $170 \mathrm{kDa}$ proteins determined by SDS-PAGE zymograms. This mRNA, reported as CDNA in the sequencing output, was a $3146 \mathrm{bp}$ transcript containing an open reading frame of 735 codons that was translated into a protein of 735 amino acids by the NCBI Orf finder algorithm (https:/ / www.ncbi.nlm.nih.gov/orffinder/) (accessed on 8 November 2021) (Table 1).

(2) The hypothetical protein coded by transcript 473 was processed for Gene Ontology Enrichment analysis online at http:/ / geneontology.org/docs/go-enrichment-analysis/ accessed on 8 November 2021 (GO:0016021 number), and this enzyme was described as an integral component of the membrane with a probable role of this type of enzyme in a drug metabolic pathway. Additionally, a K03927 KEGG number was obtained at www.genome.jp/dbget-bin/ accessed on 8 November 2021, which describes this enzyme as a carboxylesterase EC:3.1.1.1, 3.1.1.84 and 3.1.1.56, and the results are summarized in Table 1.

(3) Further bioinformatics results were obtained when the amino acid sequence was analyzed online through the InterPro algorithm (www.ebi.ac.uk/interpro/ accessed on 8 November 2021), which found that the protein exhibited several structural and functional domains, such as a noncytoplasmic carboxylesterase domain at amino acids 97-681, a transmembrane helix at amino acids 73-93 and a cytoplasmic membrane at amino acids 1-73 (Figure 3). Additionally, by comparison to similar enzymes, InterPro predicts a homodimer configuration when attached to membranes (Figure 3).

(4) Additional online analysis of the amino acid sequence at www.Bioinformatics.org accessed on 8 November 2021 showed that the predicted size of this protein is $83.5 \mathrm{kDa}$ when in a monomeric configuration and $167 \mathrm{kDa}$ when a dimer complex attached to the membrane is formed (Figure 2).

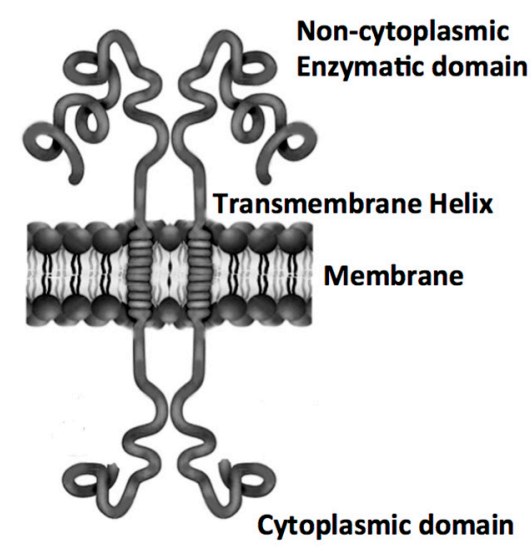

(a)

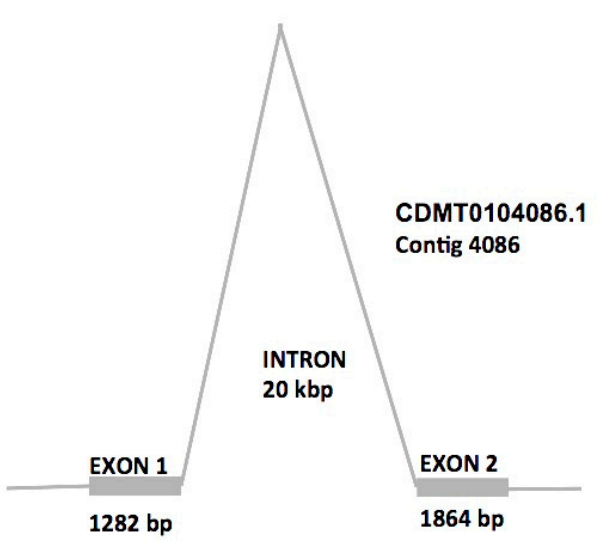

(b)

Figure 3. Bioinformatics predicted polypeptide domains (a) and exons-introns analysis (b). The Interpro algorithm predicted a non-cytoplasmic carboxylesterase domain, a trans-membrane helix domain and a cytoplasmic domain. The transcript shows identity against the GENBANK Fasciola hepatica complete genome CDMT01004086.1 contig 4086 showing two exons of 1282 and $1864 \mathrm{bp}$ respectively, and an unusually large intron of $20 \mathrm{kbp}$.

\subsection{Phylogenetic Analysis}

(1) The resulting phylogenetic tree obtained by running pBlast on transcript 473 clearly identifies two major clades with a perfect distinction between cestodes and trematodes; notably the Fasciola genus within the trematode clade was set apart in a distinctive sub- 
clade of its own, where transcript 473 carboxylesterase B, GenBank entry MT843326 can be located with a $99.7 \%$ identity with the same protein reported previously and independently at the GenBank, but labeled as hypothetical Genbank entry D915_000180. Also, within the Fasciola genus subclade is the equivalent hypothetical protein in F. gigantica with a $98 \%$ identity. An Esterase F4 of the lepidopteron Galleria mellonela was included as an out-group for enhanced accuracy of the phylogeny analysis (Figure 4).

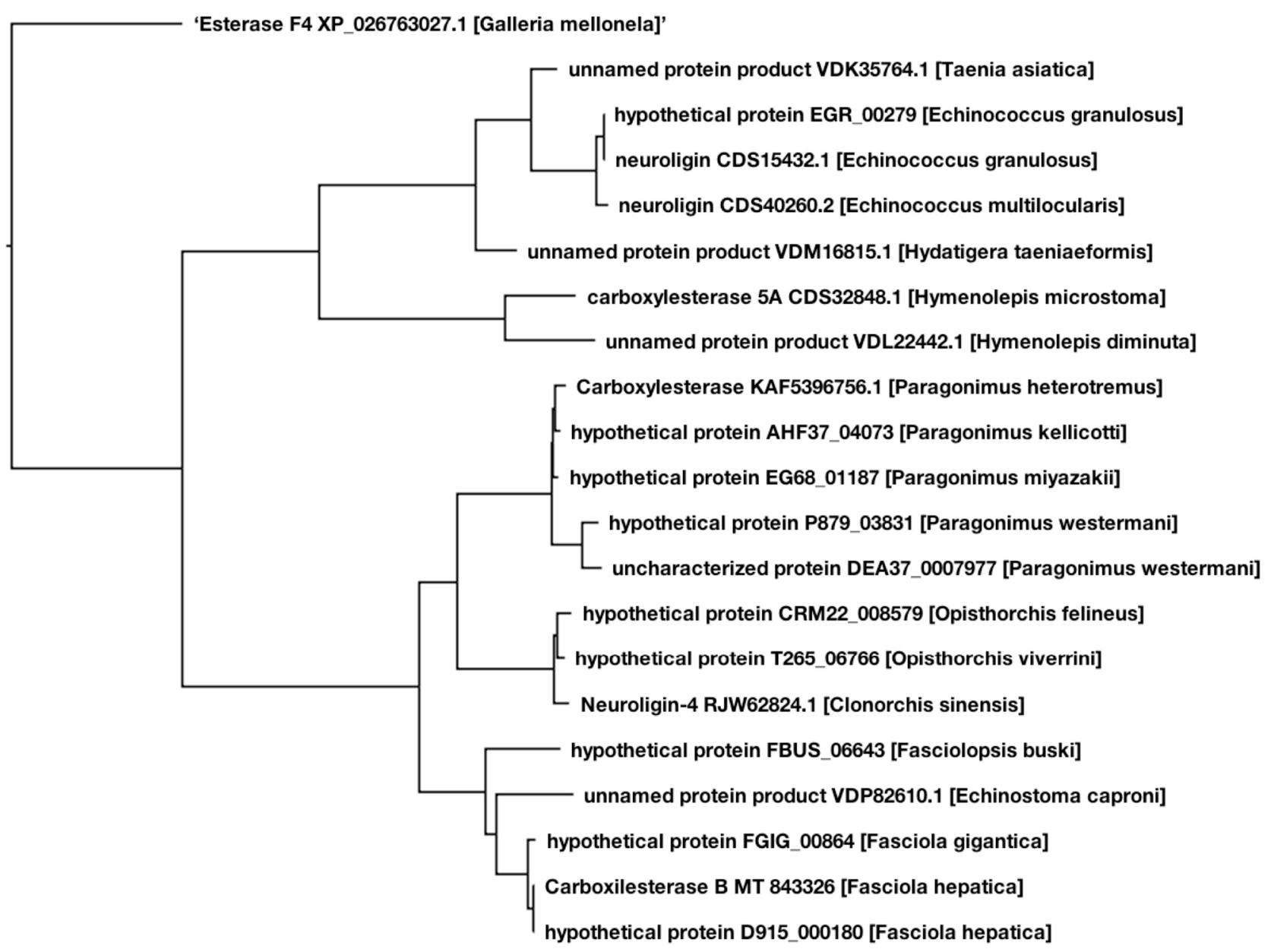

Figure 4. Carboxylesterase B deduced cDNA and amino acid sequence was submitted to the GENBANK assigning the entry number MT843326, the analysis of this sequence by pBlast algorithm at NCBI web site (blast.ncbi.nlm.nih.gov accessed on 8 November 2021) against the non-redundant protein sequences bank. Those protein sequences with an identity score above 30\%, were selected for phylogenetic analysis offered by the pBlast Tree View (www.ncbi.nlm.nih.gov/blast/treeview/ accessed on 8 November 2021) by the method of Fast Minimum Evolution [19], generating an un-rooted tree with a maximum sequence difference of 0.85 .

\section{Discussion}

The carboxylesterase of Fasciola hepatica analyzed in our study was first reported during a previous zymogram study on cytosolic protein extracts from liver flukes obtained from experimentally parasitized sheep. It was described as a $170 \mathrm{kDa}$ enzyme exhibiting high levels of catalytic activity on synthetic substrates [17]. During this study, we corroborated the presence of high levels of carboxylesterase enzymatic specific activity at $170 \mathrm{kDa}$ in both protein extracts analyzed (Figure 1), as well as an $85 \mathrm{kDa}$ carboxylesterase enzyme not previously reported. It is our interpretation that both enzymes found in the zymogram analysis are actually the same protein showing a monomeric molecular mass of $85 \mathrm{kDa}$ and a homodimeric form of $170 \mathrm{kDa}$, with both enzymes exhibiting carboxylesterase activity on synthetic chromogenic carboxylester substrates, as demonstrated by zymographic analysis 
(Figure 1). A more profound interpretation of our zymogram analysis suggests that the $170 \mathrm{kDa}$ carboxylesterase is found in the membrane-bound fraction of proteins, suggesting that this enzyme is a membrane-bound enzyme usually found as a homodimer of $170 \mathrm{kDa}$, a property common to carboxylesterases in other species $[7,20]$ (Figures 1 and 3).

(1) Since we obtained total mRNA destined for high-throughput transcriptome sequencing from this sample of parasites exhibiting high levels of carboxylesterase-specific enzymatic activity, we assumed that the expression levels of carboxylesterase 85 and $170 \mathrm{kDa}$ isozymes would show a high level of expression with a high value in FMPKs when compared to other carboxylesterases within the same transcriptome. For this purpose, we used the biochemical information obtained from the zymogram interpretation to locate the mRNA sequence of the gene in our transcriptome that could code for a transcript labeled as carboxylesterase that may show two different isoforms consistent with the protein masses and predicted enzymatic specific activity found by SDS-PAGE zymograms.

Among the ten transcripts found in the transcriptome labeled as esterase, three showed a differential expression level close to the constitutive gene Beta-Tubulin transcript 5488, showing an expression level of 19.1 FPMKs in our transcriptome (Figure 2); this transcript was used as a high expression level comparative reference within our transcriptome (Figure 2). Transcript 473 exhibited an expression level of 14.45 FPMK and predicted carboxylesterase activity by gene ontology; the closest transcripts were 4519, showing an expression of 4.11 FPMK, and transcripts 247 and 4520 with 2.6 and 2.1 FPMK, respectively. All three hypothetical proteins showed a predicted enzymatic function as cholinesterase according to the Gene Ontology analysis (Table 1) (Figure 2). All remaining transcripts labeled esterase were discarded as possible candidates due to a low level of expression and/or a predicted incompatible mRNA size or incompatible protein mass when translated.

(2) Although transcripts 247,4519 and 4520 were considered acceptable expression candidates, their predicted role as cholinesterase makes them incompatible with the enzymatic experimental conditions used during the zymogram assays; nevertheless, the transcripts were further assessed for the predicted number of amino acids when translated as well as the total mass of the expected protein; however, none of them originated an $85 \mathrm{kDa}$ mass protein by the Protein Molecular Weight Calculator algorithm. Only transcript 473 was predicted to translate into a 735 amino acid protein that was used for prediction of protein mass at Bioinformatics.org, obtaining an $83.5 \mathrm{kDa}$ protein for a monomer and $167 \mathrm{kDa}$ for a dimeric complex, almost perfectly matching our experimental calculations by zymograms. The transcript 473 sequence was submitted to NCBI pBlast analysis, and 99.7\% identity was found to mRNA 2822 of the Fasciola hepatica complete reference genome uploaded to GenBank entry No. LN627097.1, which indicates that this carboxylesterase transcript, although described as a hypothetical protein, has been available for some time. In an additional pBlast against other Fasciola hepatica complete genomes, GenBank entries CDMT01004086.1 contig 4086 and OMOY1058219.1 contig 58221 exhibit complete identity in two different regions of both genomes separated in both cases by a DNA space of approximately $20 \mathrm{kbp}$, these data are consistent with the presence of two exons of 1282 and $1864 \mathrm{bp}$ separated by an unusually large intron of $20 \mathrm{kbp}$ (Figure 3).

(3) The InterPro and Phyre 2 algorithms found a noncytoplasmic, extracellular carboxylesterase B domain spanning amino acids 97 to 681 as well as a transmembrane helix at amino acids 73 to 93 and a cytoplasmic domain at positions 1 to 73 , which corroborates that this enzyme is embedded in the cellular membrane as an integral part of it. Gen Ontology Enrichment Analysis at Genontology.org [21], concurs with this assessment and assigns number GO:0016021 to this sequence, predicting that the F. hepatica carboxylesterase plays a role as an integral component of the membrane and further suggests that it may be part of the cytochrome $\mathrm{bo}_{3}$ ubiquinol oxidase system pathway. The probable function of this carboxylesterase in drug metabolism is independently suggested by KEGG orthology analysis at genome.jp [22,23], assigning KEGG No. K03927, which describes this protein as carboxylesterase type 2 (EC:3.1.1.1 3.1.1.84 3.1.1.56) and suggests a probable role in drug metabolism pathways in conjunction with other enzymes. Both GO and KEGG num- 
bers are compatible with our experimental analysis. Since most carboxylesterase-specific activity has been found to bind to the membrane fraction as a homodimer complex, experimental evidence is compatible with an enzyme working as an integral component of the cellular membrane (Figure 3). The enzymatic activity found in the soluble fraction is consistent with a disrupted monomeric enzyme produced during the preparation of parasite protein extracts.

The mRNA transcript now converted to cDNA was submitted to GenBank, where it was assigned the entry number MT843326, and it can be directly analyzed by pBlast against the nonredundant protein dataset $[24,25]$. The results are shown in the phylogenetic tree displayed in Figure 4. The 20 highest identities were obtained against parasitic flatworm proteins, and only a handful of these sequences have been correctly described as carboxylesterases or esterase-like proteins, such as carboxylesterase $5 \mathrm{~A}$ of the cestode Hymenolepis microstoma, a carboxylesterase of the trematode Paragonimus heterotremus, and three neuroligins, nonenzymatic proteins closely related to cholinesterase, in Echinococcus granulosus, E. multilucularis and Clonorchis sinensis. The rest of the highest identity proteins were either hypothetical or unnamed proteins of flat worms (Figure 3). The resulting phylogenetic tree obtained by the pBlast toolkit clearly identifies two major clades with a perfect distinction between cestodes and trematodes; notably, the Fasciola genus within the trematode clade was set apart in a distinctive subclade of its own, where our carboxylesterase B, GenBank entry MT843326, can be located with $99.7 \%$ identity to the same protein reported previously and independently in GenBank, but labeled as hypothetical. Additionally, within the Fasciola genus subclade is the equivalent hypothetical protein in Fasciola gigantica with 98\% identity. Esterase F4 of the lepidopteron Galleria mellonela was included as an out-group for the enhanced accuracy of the phylogenetic analysis (Figure 4).

The bioinformatics analysis of transcript 473 did not find a direct biochemical pathway that may demonstrate an enzymatic effect on benzimidazole-derived anthelmintics; this does not mean that the carboxylesterase B does not interact with secondary metabolites, our study suggests that this enzyme may be part of several metabolic pathways that are implicated in the metabolism of drugs and their metabolites. We already know that triclabendazole anthelmintics have been designed to be metabolically transformed to a more toxic molecule for parasitic helminths [17]; therefore, we are not looking for the catalytic properties of carboxylesterase B on known chemical structures of anthelmintics. Instead, we are looking at the catalytic properties of carboxylesterase B on secondary metabolites of benzimidazole-derived anthelmintics, and our study identified several metabolic pathways that may yield a probable substrate for carboxylesterase B yet to be detected.

\section{Materials and Methods}

\subsection{Animals and the Parasite Strain}

Animal management was performed according to the ethical guidelines of our institutions, and animal use was performed according to the Mexican norm NOM-062-ZOO-1999, and its technical specifications for the production, care and use of laboratory animals. The F. hepatica reference strain was maintained as an anthelmintic bioassay reference at the National Center for Disciplinary Research in Veterinary Parasitology (INIFAP, Jiutepec, Morelos, Mexico) and previously registered as NCBI-BioSample SAMN16822856 [3], 90 metacercariae were orally inoculated in a six-months-old rabbit. Parasitic development was confirmed four months later by the presence of liver fluke eggs in the feces. The rabbit was sacrificed, and the liver was removed and dissected. The parasites were recovered from the liver, and a group of twenty parasites was collected in $20 \mathrm{~mL}$ of Minimal Essential Medium Eagle (MEM, Sigma Chemicals, St. Louis, MO, USA) supplemented with 10\% fetal bovine serum (FBS GIBCO ). 


\subsection{Enzyme Analysis}

For protein extraction purposes, a sample of five parasites was washed in phosphate buffered saline (PBS) at a $\mathrm{pH}$ of 7.2 , frozen at $-196{ }^{\circ} \mathrm{C}$, and macerated into a fine powder according to a previously published procedure [26]. The macerated samples were homogenized (1:1) in PBS at a pH of 7.2, and centrifuged at $5000 \times g$ for $5 \mathrm{~min}$, and the resulting supernatant was considered crude extract for carboxylesterase-specific activity purposes. The crude extract was centrifuged at $20,000 \times g$ for $30 \mathrm{~min}$. The resulting pellets were considered the insoluble membrane fraction, and the supernatant was considered the soluble cytosol fraction. All fractions were collected for determination of the protein content using the Lowry method with bovine serum albumin as a standard [27], The protein extracts were stored at $-80^{\circ} \mathrm{C}$ until assayed. The protein samples were used to determine the carboxylesterase enzymatic activity in F. hepatica using $\alpha$-naphthyl acetate coupled to the diazonium salt Fast Gardner (Sigma Chemicals, MO, USA) as a previously reported substrate [28] on $100 \mu \mathrm{g}$ of protein extracts from each sample assayed with four replications. The amount of $\alpha$-naphthol-Azo dye released by the enzymatic reaction was measured spectrophotometrically at $524 \mathrm{~nm}$. Absorbance values were converted to micromoles of hydrolyzed substrate/minute/milligram of protein according to a previously described method [28].

SDS-PAGE was performed on $12.5 \%$ gels prepared according to a previously reported method [29]. A commercial prestained protein mass standard (Thermo-Fisher, Walthman, MA, USA) was used as a mass reference, and Lab4 ${ }^{\circledR}$ algorithm molecular mass calibration was performed. Five micrograms of $F$. hepatica from each protein extract from the parasites was loaded on each well. SDS-PAGE was applied at an electric current of 100 volts for $90 \mathrm{~min}$, and afterward it was processed for carboxyl esterase identification using the method of $\alpha$-naphthyl acetate coupled to the diazonium salt Fast Gardner as a previously reported substrate [20]. The presence of carboxylesterase bands was recorded in digital images and used to estimate the isozyme molecular masses using the Lab4 ${ }^{\circledR}$ algorithm.

\subsection{High throughput mRNA Sequencing}

(1) RNA extraction. Five parasites were frozen at $-196^{\circ} \mathrm{C}$ and macerated into a fine powder; RNA was extracted using the phenol-chloroform procedure as described previously [29]. RNA quality, integrity, 28S/18S RNA ratio and fragment length distribution, were assessed by capillary electrophoresis using an Agilent 2100 Bioanalyzer. For estimation of the total RNA sample QC such as the RNA concentration and RIN value, a Nanodrop ${ }^{\circledR}$ was used as previously described [3].

(2) Transcriptome sequencing was carried out by the BGI Genomics Co. Ltd. Using the Illumina HiSeq 4000 platform. For transcriptome assembly the F. hepatica reference genome GCA_900302435.1 was used for transcriptome assembly and differential expression differential display in FPMK. The BLAST similarity analysis was performed using the assembled transcriptome; Gene Ontology was performed according to a previously reported method [21], and KEGG orthology terms associated with the transcripts were extracted from the UniProt, Phyre2 and WormBase-ParaSite databases, integrated with the BLAST search results by the sequencer according to previous reports $[22,23,30]$ and delivered as part of the sequence report. In a comprehensive search for $F$. hepatica esterase DNA sequences within the complete F. hepatica genomes reported in Bioprojects PRJEB6687, PRJEB25283 and PRJNA179522 as well as in the complete transcriptome reported in Bioproject PRJNA330752 using WormBase Parasite as well as GenBank search toolkits as previously described [24,25], every esterase found in the databases was searched for similar sequences in our transcriptome by the BLAST similarity analysis according to a previous report [31]. The obtained F. hepatica transcriptome database was made public at GenBank BioProject PRJNA649302, BioSample SAMN16822856, RNAseq data SRR13076126 (https: / / trace.ncbi.nlm.nih.gov/Traces/sra/?run=SRR13076124) and previously described elsewhere [3]. 


\subsection{Phylogenetic Analysis}

The deduced carboxylesterase $\mathrm{B}$ cDNA and amino acid sequence were submitted to the GenBank, assigning the entry number MT843326. The analysis of this sequence by the pBlast algorithm at the NCBI website (blast.ncbi.nlm.nih.gov accessed on 8 November 2021) against the nonredundant protein sequences bank was performed according to a previous report [31]. Those protein sequences with an identity score above $30 \%$ were selected for phylogenetic analysis offered by the pBlast Tree View (www.ncbi.nlm.nih.gov/blast/ treeview / accessed on 8 November 2021) by the method of fast minimum evolution [19], generating an unrooted tree with a maximum sequence difference of 0.85 .

\section{Conclusions}

Our study suggests that the $F$. hepatica carboxylesterase B is an essential enzyme for the metabolism of the liver fluke; our bioinformatics analysis of this gene showed that the enzyme is highly conserved in parasitic flatworms, suggesting a fundamental role in parasitic helminth physiology. The literature inquiry describes these enzymes as part of the xenobiotic metabolizing group of enzymes, and some of them may be involved in drug metabolism [9] our bioinformatics study confirms this assessment and suggests several metabolic pathways that need further studies. The carboxylesterase studied here was discovered to be induced by the presence of benzamidine-derived anthelmintics in parasitized sheep hosts [17], and our study confirmed its presence and elaborates on the possible role in drug metabolism. Our bioinformatics analysis further suggest that this $85 \mathrm{kDa}$ enzyme works as a $170 \mathrm{kDa}$ dimer complex as an integral component of the cellular membrane interacting with several other enzymes [8], some of them known XMEs. Our study shows that the identified $3146 \mathrm{bp}$ mRNA transcript coding a $2205 \mathrm{bp}$ open reading frame translates into a protein of 735 amino acids that has a putative carboxylesterase $B$ functional enzymatic activity undisclosed until now; the gene coding for this enzyme was found to be highly conserved in other reported complete F. hepatica genomes and suggests that this enzyme plays an important role in other flatworms. However, until now, no evidence of the functionality of these putative genes existed, and they were labeled hypothetical proteins. The carboxylesterase B activity can be assayed on synthetic chromogenic substrates, something useful for biochemical testing in follow-up studies. Additionally, our study now makes DNA data for this carboxylesterase available to the scientific community for follow up studies.

Author Contributions: Y.J.P.-G.: Experimental execution. E.M.-M.: Conceptualization, Methodology, Writing-original draft preparation. R.C.-B.: Samples Processing, Writing-Original draft preparation. H.A.-D.: Analysis, Investigation. S.S.: Analysis, Investigation. E.R.: Samples Processing, Analysis, and Investigation. V.N.-P.: Writing-Reviewing and Analysis. M.d.R.S.-C.: Analysis, Investigation. All authors have read and agreed to the published version of the manuscript.

Funding: This research received funding from the National Council for Science and Technology of Mexico (CONACYT) grants CIPN 2015-021, 255478 and by funds from INIFAP 13174433989, 1172635360, DGAPA/UNAM PAPIIT-IN204214, PAPIIT-IN206517.

Institutional Review Board Statement: Animal management was performed accordingly to the ethical guidelines of our institutions. Animal care and use was according to the Mexican norm NOM-062-ZOO-1999, and its technical specifications for production, care and use of laboratory animals and supervised by the laboratory animals handling ethics committee.

Data Availability Statement: Access to RNAseq data Transcriptome Adult TCBZ resistant, Tanscriptome dataset is available at https: / / www.ncbi.nlm.nih.gov / sra/SRX9523046 accessed on 8 November 2021: RNAseq data Transcriptome Adult TCBZ resistant: https:/ / www.ncbi.nlm.nih.gov/sra/SRX9 523047[accn] accessed on 8 November 2021., https:/ / www.ncbi.nlm.nih.gov/biosample/16822857 accessed on 8 November 2021. NCBI-BioSample SAMN16822858 (Adult Fasciola hepatica Triclabendazole resistant). RNAseq data SRR13076124 available on-line at https:/ / trace.ncbi.nlm.nih.gov / Traces /sra/?run=SRR13076124 accessed on 8 November 2021. and tables that includes differential expression for each transcript in FPKM, GO and KEGG numbers as well as GENBANK accession 
numbers for each sequence, details of raw read generated, assembly and annotation information, overall transcriptomic annotation information such as mapping rate, number of known and unknown transcript identified, splicing events and long noncoding RNA transcripts as well as the annotated gene ontology divided in number of genes found as cellular components or fulfilling a biological process or molecular function.

Conflicts of Interest: The authors declare that they have no known competing financial interests or personal relationships, which have, or could be perceived to have, influenced the work reported in this article.

\section{References}

1. Zumaquero-Ríos, J.L.; Sarracent-Pérez, J.; Rojas-García, R.; Rojas-Rivero, L.; Martínez-Tovilla, Y.; Valero, M.A.; Mas-Coma, S. Fascioliasis and Intestinal Parasitoses Affecting Schoolchildren in Atlixco, Puebla State, Mexico: Epidemiology and Treatment with Nitazoxanide. PLoS Negl. Trop. Dis. 2013, 7, e2553. [CrossRef] [PubMed]

2. Bargues, M.D.; Artigas, P.; Khoubbane, M.; Mas-Coma, S. DNA Sequence Characterisation and Phylogeography of Lymnaea Cousini and Related Species, Vectors of Fascioliasis in Northern Andean Countries, with Description of L. meridensis n. Sp. (Gastropoda: Lymnaeidae). Parasites Vectors 2011, 4, 132. [CrossRef] [PubMed]

3. Miranda-Miranda, E.; Cossio-Bayugar, R.; Aguilar Díaz, H.; Narváez Padilla, V.; Sachman-Ruiz, B.; Reynaud, E. Transcriptome Assembly Dataset of Anthelmintic Response in Fasciola hepatica. Data Brief 2021, 35, 106808. [CrossRef] [PubMed]

4. Boyko, K.M.; Kryukova, M.V.; Petrovskaya, L.E.; Nikolaeva, A.Y.; Korzhenevsky, D.A.; Novototskaya-Vlasova, K.A.; Rivkina, E.M.; Dolgikh, D.A.; Kirpichnikov, M.P.; Popov, V.O. Crystal Structure of PMGL2 Esterase from the Hormone-Sensitive Lipase Family with GCSAG Motif around the Catalytic Serine. PLoS ONE 2020, 15, e0226838. [CrossRef]

5. Boyko, K.M.; Kryukova, M.V.; Petrovskaya, L.E.; Kryukova, E.A.; Nikolaeva, A.Y.; Korzhenevsky, D.A.; Lomakina, G.Y.; Novototskaya-Vlasova, K.A.; Rivkina, E.M.; Dolgikh, D.A.; et al. Structural and Biochemical Characterization of a Cold-Active PMGL3 Esterase with Unusual Oligomeric Structure. Biomolecules 2021, 11, 57. [CrossRef] [PubMed]

6. Scarcella, S.; Solana, M.V.; Fernandez, V.; Lamenza, P.; Ceballos, L.; Solana, H. Increase of Gluthatione S-Transferase, Carboxyl Esterase and Carbonyl Reductase in Fasciola hepatica Recovered from Triclabendazole Treated Sheep. Mol. Biochem. Parasitol. 2013, 191, 63-65. [CrossRef]

7. Matoušková, P.; Vokřál, I.; Lamka, J.; Skálová, L. The Role of Xenobiotic-Metabolizing Enzymes in Anthelmintic Deactivation and Resistance in Helminths. Trends Parasitol. 2016, 32, 481-491. [CrossRef] [PubMed]

8. Hosokawa, M. Structure and Catalytic Properties of Carboxylesterase Isozymes Involved in Metabolic Activation of Prodrugs. Molecules 2008, 13, 412-431. [CrossRef]

9. Parkinson, A. Biotransformation of xenobiotics. In Cassarett and Doulls Toxicology: The Basic Science of Poisons; McGraw-Hill Medical: New York, NY, USA, 1996; pp. 113-196.

10. Kontogiannatos, D.; Swevers, L.; Maenaka, K.; Park, E.Y.; Iatrou, K.; Kourti, A. Functional Characterization of a Juvenile Hormone Esterase Related Gene in the Moth Sesamia nonagrioides through RNA Interference. PLoS ONE 2013, 8, e73834. [CrossRef]

11. Istvan, E.S.; Mallari, J.P.; Corey, V.C.; Dharia, N.V.; Marshall, G.R.; Winzeler, E.A.; Goldberg, D.E. Esterase Mutation Is a Mechanism of Resistance to Antimalarial Compounds. Nat. Commun. 2017, 8, 14240. [CrossRef] [PubMed]

12. Butler, J.H.; Baptista, R.P.; Valenciano, A.L.; Zhou, B.; Kissinger, J.C.; Tumwebaze, P.K.; Rosenthal, P.J.; Cooper, R.A.; Yue, J.-M.; Cassera, M.B. Resistance to Some But Not Other Dimeric Lindenane Sesquiterpenoid Esters Is Mediated by Mutations in a Plasmodium falciparum Esterase. ACS Infect. Dis. 2020, 6, 2994-3003. [CrossRef] [PubMed]

13. Dahan-Moss, Y.L.; Koekemoer, L.L. Analysis of Esterase Enzyme Activity in Adults of the Major Malaria Vector Anopheles funestus. Parasites Vectors 2016, 9, 110. [CrossRef]

14. Schama, R.; Pedrini, N.; Juárez, M.P.; Nelson, D.R.; Torres, A.Q.; Valle, D.; Mesquita, R.D. Rhodnius Prolixus Supergene Families of Enzymes Potentially Associated with Insecticide Resistance. Insect Biochem. Mol. Biol. 2016, 69, 91-104. [CrossRef] [PubMed]

15. Wang, L.-L.; Huang, Y.; Lu, X.-P.; Jiang, X.-Z.; Smagghe, G.; Feng, Z.-J.; Yuan, G.-R.; Wei, D.; Wang, J.-J. Overexpression of Two $\alpha$-Esterase Genes Mediates Metabolic Resistance to Malathion in the Oriental Fruit Fly, Bactrocera dorsalis (Hendel). Insect Mol. Biol. 2015, 24, 467-479. [CrossRef]

16. Shin, D.; Smartt, C.T. Assessment of Esterase Gene Expression as a Risk Marker for Insecticide Resistance in Florida Culex nigripalpus (Diptera: Culicidae). J. Vector Ecol. 2016, 41, 63-71. [CrossRef] [PubMed]

17. Scarcella, S.; Miranda-Miranda, E.; Cossío-Bayúgar, R.; Ceballos, L.; Fernandez, V.; Solana, H. Increase of Carboxylesterase Activity in Fasciola hepatica Recovered from Triclabendazole Treated Sheep. Mol. Biochem. Parasitol. 2012, 185, 151-153. [CrossRef] [PubMed]

18. Beesley, N.J.; Williams, D.J.L.; Paterson, S.; Hodgkinson, J. Fasciola hepatica Demonstrates High Levels of Genetic Diversity, a Lack of Population Structure and High Gene Flow: Possible Implications for Drug Resistance. Int. J. Parasitol. 2017, 47, 11-20. [CrossRef]

19. Desper, R. Theoretical Foundation of the Balanced Minimum Evolution Method of Phylogenetic Inference and Its Relationship to Weighted Least-Squares Tree Fitting. Mol. Biol. Evol. 2003, 21, 587-598. [CrossRef] [PubMed]

20. Manchenko, G.P. Handbook of Detection of Enzymes on Electrophoretic Gels, 2nd ed.; CRC Press LLC: Boca Raton, FL, USA, 2003. 
21. Mi, H.; Muruganujan, A.; Casagrande, J.T.; Thomas, P.D. Large-Scale Gene Function Analysis with the PANTHER Classification System. Nat. Protoc. 2013, 8, 1551-1566. [CrossRef] [PubMed]

22. Kanehisa, M.; Sato, Y.; Morishima, K. BlastKOALA and GhostKOALA: KEGG Tools for Functional Characterization of Genome and Metagenome Sequences. J. Mol. Biol. 2016, 428, 726-731. [CrossRef] [PubMed]

23. Kanehisa, M.; Sato, Y.; Kawashima, M.; Furumichi, M.; Tanabe, M. KEGG as a Reference Resource for Gene and Protein Annotation. Nucleic Acids Res. 2016, 44, D457-D462. [CrossRef] [PubMed]

24. Howe, K.L.; Bolt, B.J.; Cain, S.; Chan, J.; Chen, W.J.; Davis, P.; Done, J.; Down, T.; Gao, S.; Grove, C.; et al. WormBase 2016: Expanding to Enable Helminth Genomic Research. Nucleic Acids Res. 2016, 44, D774-D780. [CrossRef] [PubMed]

25. Howe, K.L.; Bolt, B.J.; Shafie, M.; Kersey, P.; Berriman, M. WormBase ParaSite-A Comprehensive Resource for Helminth Genomics. Mol. Biochem. Parasitol. 2017, 215, 2-10. [CrossRef]

26. Ericsson, C.; Franzén, B.; Nistér, M. Frozen Tissue Biobanks. Tissue Handling, Cryopreservation, Extraction, and Use for Proteomic Analysis. Acta Oncol. 2006, 45, 643-661. [CrossRef] [PubMed]

27. Peterson, G.L. A Simplification of the Protein Assay Method of Lowry et al. Which Is More Generally Applicable. Anal. Biochem. 1977, 83, 346-356. [CrossRef]

28. Bardi, L.; Dell'oro, V.; Delfini, C.; Marzona, M. A Rapid Spectrophotometric Method to Determine Esterase Activity of Yeast Cells in an Aqueous Medium. J. Inst. Brew. 1993, 99, 385-388. [CrossRef]

29. Sambrook, J.; Russell, D.W. Molecular Cloning: A Laboratory Manual, 3rd ed.; Cold Spring Harbor Laboratory Press: Cold Spring Harbor, NY, USA, 2001; ISBN 978-0-87969-577-4.

30. Kelley, L.; Mezulis, S.; Yates, C.; Wass, M.N.; Sternberg, M.J.E. The Phyre2 Web Portal for Protein Modeling, Prediction and Analysis I Nature Protocols. Nat. Protoc. 2015, 10, 845-858. [CrossRef] [PubMed]

31. Boratyn, G.M.; Schäffer, A.A.; Agarwala, R.; Altschul, S.F.; Lipman, D.J.; Madden, T.L. Domain Enhanced Lookup Time Accelerated BLAST. Biol. Direct 2012, 7, 12. [CrossRef] 